Meta

Journal des traducteurs

Translators' Journal

\title{
Quels exemples (lexicographiques) pour le traducteur ?
}

\section{Clémence Belleflamme}

Volume 64, numéro 1, avril 2019

URI : https://id.erudit.org/iderudit/1065326ar

DOI : https://doi.org/10.7202/1065326ar

Aller au sommaire du numéro

\section{Éditeur(s)}

Les Presses de l’Université de Montréal

\section{ISSN}

0026-0452 (imprimé)

1492-1421 (numérique)

Découvrir la revue

Citer cet article

Belleflamme, C. (2019). Quels exemples (lexicographiques) pour le traducteur? Meta, 64(1), 3-19. https://doi.org/10.7202/1065326ar

\section{Résumé de l'article}

Malgré ses limitations, le dictionnaire bilingue reste l'outil privilégié des étudiants lorsqu'ils effectuent une tâche de traduction vers leur langue maternelle. Ils le consultent principalement pour comprendre un mot ou une expression, en vérifier le sens et chercher un équivalent adéquat, mais ne tirent pas toujours entièrement profit des différents éléments de microstructure, tels que les exemples d'usage. Si les fonctions sémantiques, grammaticales ou même stylistiques de l'exemple sont bien connues, ses fonctions de traduction sont aujourd'hui encore assez peu mises en évidence. Or, elles s'avèrent particulièrement utiles pour les jeunes traducteurs. Dans cet article, nous communiquons les résultats de l'analyse d'exemples issus de trois dictionnaires bilingues allemand-français, reposant sur l'examen des divergences que présentent les exemples lexicographiques et leur traduction. L'objectif poursuivi était de mieux cerner quel type d'exemple est le plus utile à l'apprenti traducteur. Pour atteindre cet objectif, nous avons exploré deux pistes principales. D’une part, nous avons cherché à repérer les difficultés de linguistique contrastive illustrées par les exemples lexicographiques et leur traduction, ainsi qu'à déterminer quelles solutions les dictionnaires apportaient à l'utilisateur. D'autre part, l'utilité d'un dictionnaire étant directement liée à la qualité des traductions fournies, nous nous sommes penchée sur certaines traductions discutables afin de suggérer des pistes d'amélioration pour un dictionnaire à destination des jeunes traducteurs.
Ce document est protégé par la loi sur le droit d'auteur. L’utilisation des services d'Érudit (y compris la reproduction) est assujettie à sa politique d'utilisation que vous pouvez consulter en ligne.

https://apropos.erudit.org/fr/usagers/politique-dutilisation/ 


\title{
Quels exemples (lexicographiques) pour le traducteur?
}

\author{
CLÉMENCE BELLEFLAMME \\ Université de Liège, Liège, Belgique \\ c.belleflamme@uliege.be
}

\begin{abstract}
RÉSUMÉ
Malgré ses limitations, le dictionnaire bilingue reste l'outil privilégié des étudiants lorsqu'ils effectuent une tâche de traduction vers leur langue maternelle. Ils le consultent principalement pour comprendre un mot ou une expression, en vérifier le sens et chercher un équivalent adéquat, mais ne tirent pas toujours entièrement profit des différents éléments de microstructure, tels que les exemples d'usage. Si les fonctions sémantiques, grammaticales ou même stylistiques de l'exemple sont bien connues, ses fonctions de traduction sont aujourd'hui encore assez peu mises en évidence. Or, elles s'avèrent particulièrement utiles pour les jeunes traducteurs. Dans cet article, nous communiquons les résultats de l'analyse d'exemples issus de trois dictionnaires bilingues allemand-français, reposant sur l'examen des divergences que présentent les exemples lexicographiques et leur traduction. L'objectif poursuivi était de mieux cerner quel type d'exemple est le plus utile à l'apprenti traducteur. Pour atteindre cet objectif, nous avons exploré deux pistes principales. D'une part, nous avons cherché à repérer les difficultés de linguistique contrastive illustrées par les exemples lexicographiques et leur traduction, ainsi qu'à déterminer quelles solutions les dictionnaires apportaient à l'utilisateur. D'autre part, l'utilité d'un dictionnaire étant directement liée à la qualité des traductions fournies, nous nous sommes penchée sur certaines traductions discutables afin de suggérer des pistes d'amélioration pour un dictionnaire à destination des jeunes traducteurs.
\end{abstract}

\section{ABSTRACT}

Despite its limitations, the bilingual dictionary remains students' favourite tool when confronted with a translation task into their mother tongue. They refer to it mostly to understand a word or an expression, to check its meaning and to find an adequate equivalent, however, they do not always take full advantage of the different microstructural elements, such as usage examples. While the semantic, grammatical or stylistic functions of the example are well-known, their translational functions are still rarely valued and acknowledged. Yet they have proven very useful to novice translators. In this article, I shall analyse examples taken from three German-French bilingual dictionaries. The analysis is based on the divergences between the lexicographical examples and their translations. The aim was to investigate which type of example turns out to be the most useful to novice translators. To reach this objective, I pursue two main lines of reflection. On the one hand, I seek to identify the contrastive linguistic issues raised by the lexicographical examples and their translations, and to determine which solutions the dictionaries offered to the users. On the other hand, as a bilingual dictionary's usefulness is directly related to the quality of the translations provided, I examine certain questionable translations in order to suggest possible ways to improve dictionaries intended for novice translators.

\section{RESUMEN}

A pesar de sus limitaciones, el diccionario bilingüe sigue siendo la herramienta privilegiada de los estudiantes a la hora de realizar una tarea de traducción a su lengua materna. Lo consultan principalmente para entender una palabra o una expresión, para comprobar 
su significado y para buscar un equivalente adecuado, pero no siempre sacan provecho de los distintos elementos de microestructura, como los ejemplos de uso. Si bien las funciones semánticas, gramaticales e incluso estilísticas del ejemplo se han descrito con mucha frecuencia, sus funciones de traducción han permanecido bastante desconocidas hasta la actualidad. Sin embargo, resultan particularmente útiles para los jóvenes traductores. En este artículo, comunicamos los resultados del análisis de ejemplos extraídos de tres diccionarios bilingües alemán-francés, de acuerdo con un examen de las divergencias que presentan los ejemplos lexicográficos y su traducción. El objetivo es entender mejor qué tipo de ejemplo es de mayor utilidad para el aprendiz de traductor. Para alcanzar dicho objetivo hemos explorado dos ejes de reflexión principales. Por una parte, hemos intentado identificar los aspectos de la lingüística contrastiva ilustrados por los ejemplos lexicográficos y su traducción, así como determinar qué soluciones les proponen los diccionarios a los usuarios. Por otra parte, como la utilidad de un diccionario está estrechamente ligada a la calidad de sus traducciones, hemos observado algunas traducciones discutibles para hacer sugerencias con el propósito de mejorar la calidad de los diccionarios dedicados a jóvenes traductores.

\section{MOTS CLÉS/KEYWORDS/PALABRAS CLAVE}

métalexicographie, dictionnaire bilingue, exemple, linguistique contrastive, traduction metalexicography, bilingual dictionary, example, contrastive linguistics, translation metalexicografía, diccionario bilingüe, ejemplo, lingüística contrastiva, traducción

\section{Introduction}

Malgré ses limitations ${ }^{1}$, le dictionnaire bilingue reste l'outil privilégié des étudiants lorsqu'ils effectuent une tâche de traduction vers leur langue maternelle (Mackintosh 1998: 124; Starren et Thelen 1990: 447). Ils le consultent principalement pour comprendre un mot ou une expression, en vérifier le sens ou chercher un équivalent adéquat, mais ne tirent pas toujours entièrement profit des différents éléments de la microstructure, tels que les exemples d'usage. Si les fonctions sémantiques, grammaticales ou même stylistiques de l'exemple sont bien connues, ses fonctions de traduction sont aujourd'hui encore assez peu mises en évidence. Or, elles s'avèrent particulièrement utiles pour les jeunes traducteurs, notamment car elles sont susceptibles de leur suggérer des procédures de traduction auxquelles ils ne sont peut-être pas encore rodés (au contraire des traducteurs chevronnés).

Dans cet article, nous communiquons les résultats d'une analyse d'exemples issus de trois dictionnaires bilingues allemand-français, reposant sur un examen des divergences que présentent les exemples lexicographiques et leur traduction. L'objectif poursuivi dans le cadre de cette recherche était de mieux cerner quel type d'exemple est le plus utile à l'apprenti traducteur. Pour ce faire, nous avons exploré deux pistes principales. D’une part, l'affirmation, formulée notamment par Blanco (1995: 1153) et Humblé (2001 : 70), selon laquelle l'exemple du dictionnaire bilingue devrait attirer l'attention de l'utilisateur sur les différences existant entre les deux systèmes linguistiques en présence nous a menée à consacrer la première partie de notre travail de recherche aux grandes questions de la linguistique contrastive allemand-français. D'autre part, l'utilité d'un dictionnaire étant directement liée à la qualité des traductions fournies, nous nous sommes penchée sur certaines traductions discutables afin de suggérer des pistes d'amélioration pour un dictionnaire à destination des traducteurs.

Avant d'entrer dans l'analyse à proprement parler, nous soulèverons brièvement les questions de la définition de l'exemple lexicographique, de l'utilisation de corpus 
dans la confection de dictionnaires bilingues ainsi que des fonctions de l'exemple et, plus particulièrement, de ses fonctions de traduction. Nous exposerons ensuite les modalités de l'étude réalisée, soit les critères qui ont présidé au choix des dictionnaires analysés et la méthodologie utilisée. L'examen d'exemples significatifs constituera le cœur de cet article. Les premiers témoigneront de l'orientation linguistique de certains ouvrages et de la richesse que représentent des exemples bien choisis; les seconds mettront le doigt sur certaines lacunes des traductions et permettront de mieux cerner les enjeux de la traduction dictionnairique.

\section{Définition de l'exemple lexicographique}

Rettig (1985: 108) affirmait que l'une des tâches auxquelles la lexicographie bilingue devrait s'atteler à l'avenir était de mieux distinguer les lexèmes pluriverbaux (mehrwortige Lexeme, c'est-à-dire les collocations et locutions idiomatiques) des simples exemples d'usage. Cette question est aujourd'hui encore d'actualité puisque les auteurs ne s'accordent toujours pas sur la définition de l'exemple lexicographique.

En réalité, l'exemple est très souvent défini en fonction de la distinction entre séquences libres et séquences figées. Soit l'exemple est perçu comme une catégorie qui englobe ces deux réalités, soit il constitue l'un des pôles d'une échelle où se situent les différents types de séquences selon leur degré de figement. L'exemple est alors considéré comme la séquence la plus libre. À l'autre extrême figurent les proverbes, qui correspondent au plus haut degré de figement (Svensén 1993: 10). La collocation, combinaison de mots qui apparaissent souvent ensemble (Svensén 1993: 99), et la locution, combinaison figée dont le sens ne peut pas être déduit du sens des mots qui la composent (Svensén 1993: 108), occupent des positions intermédiaires, la collocation étant plus libre que la locution.

Selon Jacobsen, Manley et al. (1991 : 2785), il convient de distinguer le plan lexicologique du plan lexicographique. En effet, la différence entre les locutions, les collocations et les séquences libres relève de la lexicologie, alors que l'exemple, la glose et la sous-entrée sont des catégories lexicographiques. Néanmoins, ces deux plans sont liés. Sur le plan lexicologique, comme nous l'avons dit, chaque séquence peut être située sur une échelle selon son degré de figement. Or, les trois auteurs soutiennent que plus la forme est fixe, plus elle est susceptible de faire l'objet d'une sousentrée plutôt que d'un exemple. Au contraire, plus la séquence autorise la généralisation, plus l'exemple s'impose.

Jacobsen, Manley et al. (1991: 2783) plaident donc en faveur d'une définition formelle de l'exemple en tant qu'élément de microstructure, et ce, pour deux raisons. Premièrement, le terme est employé de manière trop peu rigoureuse et désigne non seulement ce que les auteurs considèrent comme de véritables exemples, soit des séquences dont l'utilisateur peut se servir comme modèle pour former de nouvelles phrases, mais aussi les locutions ou les collocations qui présentent des contextes dans lesquels le lemme pourrait être employé, mais qui ne permettent pas la généralisation. Deuxièmement, les exemples sont multifonctionnels et fournissent de manière implicite des informations que donnent déjà d'autres éléments de la microstructure (gloses, informations stylistiques, etc.). Comme il est dès lors impossible de définir l'exemple d'après une fonction qui lui serait propre, le critère de définition doit être la forme. 
Dans sa thèse intitulée El ejemplo en el diccionario bilingüe. Tipología y funciones del ejemplo en el marco de la lexicografía bilingüe general contemporánea francésespañol, español-francés, Blanco (1995) opte, lui aussi, pour une définition strictement formelle de l'exemple lexicographique:

El ejemplo en la lexicografía bilingüe general contemporánea Francés-Español, EspañolFrancés es: - un elemento opcional de la microestructura del artículo lexicográfico, caracterizado por ciertas marcas tipográficas recurrentes [...]. Presenta: - normalmente, carácter pluriverbal; - carácter multifuncional en cuanto a los distintos tipos de información lexicográfica vehiculada [...] Recibe una traducción. (Blanco 1995: 132)

Le chercheur espagnol établit une distinction entre les exemples et ce qu'il appelle les « unidades lexicográficas pluriverbales» (ULP). Celles-ci n’appartiennent pas à la microstructure, mais sont des éléments de la seconde macrostructure et, par conséquent, elles sont le sujet d'une prédication métalinguistique. Elles sont, tout comme les exemples, associées à un équivalent de traduction (Blanco 1995: 132-133). Blanco (1995) explique avoir rencontré des difficultés pratiques au moment de constituer ses bases de données, dont l'une était composée d'exemples et l'autre d'ULP. En effet, les cas ambigus sont nombreux et les dictionnaires n'adoptent pas toujours un système clair pour différencier les deux types de séquences (Blanco 1995: 135). C’est pour cette raison qu'il a finalement utilisé comme critère de sélection les marques typographiques associées à l'exemple dans chacun des dictionnaires (Blanco 1995: 134).

À l'instar de Blanco, nous avons opté pour une définition purement formelle de l'exemple en tant qu'élément de microstructure et nous nous sommes servie des distinctions établies par les dictionnaires eux-mêmes, c'est-à-dire que nous avons isolé les exemples des autres catégories d'informations grâce aux caractéristiques typographiques associées à ce type de séquence dans chacun des ouvrages.

\section{Utilisation de corpus dans la confection des dictionnaires bilingues}

Traditionnellement, les lexicographes distinguent les exemples en fonction de leur source, selon qu'ils sont construits ou cités. Les exemples construits sont imaginés par le lexicographe; leur forme dépend donc de sa compétence linguistique ou de celle d'un informant (Toope 1996: 45). Selon Martin (1989), l'exemple construit est autonyme, c'est-à-dire qu'il ne renvoie pas au monde. Il a le statut de phrase et non pas d'énoncé: "L'exemple construit, dépouillant l'énoncé de tout renvoi à une situation réelle, conduit à un artefact qui n'est que le lieu du sens» (Martin 1989: 600). Grundy (1996: 131) estime qu' " [...] un travail sur la langue qui se fait uniquement par introspection produira inévitablement un grand nombre d'exemples d'usages potentiels (par opposition à des usages réels) ». L'exemple cité est, quant à lui, issu d'un corpus. Comme l'explique Martin (1989: 600): "Certes, par sa fonction illustrative, il a lui aussi, dans l'article lexicographique, le statut d'un autonyme. Mais le texte dont il est extrait est un texte spontanément créé par un locuteur.» Il a donc une valeur philologique de témoignage (Martin 1989: 601). Aujourd'hui, l'utilisation de corpus dans la confection de dictionnaires bilingues s'est généralisée, au point qu'il est devenu inconcevable pour les lexicographes de s'en passer (Humblé 2001: 81). La proportion d'exemples cités a donc fortement augmenté.

Toutefois, la frontière entre les deux types d'exemples n'est pas si nette qu'il n'y paraît à première vue. Martin (1989: 600) écrit: «Souvent, l’exemple construit prend 
son départ à des énoncés authentiques et s'obtient par des simplifications successives qui en font un type abstrait, libéré de toute incidence situationnelle. » Un dictionnaire peut en effet être corpus-driven, corpus-based ou corpus-informed en fonction du degré d'utilisation du corpus. Tognini-Bonelli $(2001: 66,84)$ affirme que, dans un dictionnaire du type corpus-driven, les entrées reflètent directement les données du corpus, alors que dans un dictionnaire du type corpus-based, il n'est utilisé que pour corroborer des hypothèses déjà existantes avant consultation du corpus. Dans les dictionnaires du type corpus-informed, l'utilisation du corpus est encore plus limitée (Kosem 2016: 79). Le traitement réservé aux exemples diffère lui aussi: dans un dictionnaire corpus-driven, ils sont utilisés tels quels, cités directement sous la forme qu'ils ont dans le corpus; dans un dictionnaire corpus-based, ils peuvent être modifiés afin d'être plus lisibles et facilement compréhensibles; dans un dictionnaire corpus-informed, le lexicographe peut ajouter des phrases extraites du corpus (parfois modifiées) aux entrées existantes (Kosem 2016: 79). Mais le corpus n'est pas seulement un réservoir où le lexicographe puise des exemples; il s'avère également très utile pour les traduire. Lors de la phase de transfert ${ }^{2}$, le lexicographe de langue cible traduit le terme en question dans tous les contextes proposés par le lexicographe de langue source au terme de l'analyse. Après s'être fié à son intuition, il se tourne vers le corpus pour y trouver, vérifier ou corriger des traductions (Atkins 2008: 473). Il sélectionne alors l'équivalent le plus adéquat pour apparaître comme «traduction directe» de l'entrée; cet équivalent s'apparentant à ce qu'Atkins appelle une contextfree translation. Les exemples fourniraient des façons plus subtiles de traduire le terme en question, des context-sensitive translations (Atkins 2008: 467). Il est important de noter que les lexicographes peuvent recourir à deux types de corpus bilingues: les corpus de traduction, qui mettent en parallèle des textes originaux et leur traduction, et les corpus comparables, constitués uniquement de textes originaux, qui partagent certaines caractéristiques telles que le type de texte, le thème, la date de publication, etc. Si les corpus de traduction sont plus faciles à utiliser puisqu'ils fournissent des correspondances directes, les corpus comparables garantissent un accès à l'usage réel. Les lexicographes disposent donc de moyens toujours plus sûrs pour donner au lecteur des exemples (traduits) les plus fidèles possible à l'usage.

Au sein des dictionnaires, il est très difficile de savoir exactement quels exemples sont tirés directement d'un corpus et lesquels sont adaptés, quelles traductions sont effectivement attestées et lesquelles émanent du lexicographe de langue cible. Dans tous les cas, les exemples fournis et les traductions proposées se veulent représentatifs de l'usage respectif dans les deux langues en présence. Nous avons dès lors envisagé les traductions indépendamment de leur source pour déterminer quelles stratégies de traduction les dictionnaires illustraient.

\section{Fonctions de l'exemple lexicographique}

De nombreux chercheurs se sont penchés sur le rôle des exemples dans les dictionnaires bilingues. Tous mettent en rapport la fonction de l'exemple lexicographique avec le type d'activité qu'entend favoriser le dictionnaire: tâche active de production (encoding) ou tâche passive de compréhension (decoding). Alors que la plupart des dictionnaires entendent encore servir ces deux objectifs simultanément, les chercheurs s'accordent sur le fait que les dictionnaires devraient être conçus différemment 
selon le type d'activité privilégié. La différence entre decoding et encoding devrait se marquer au niveau de la forme et du contenu des dictionnaires.

Ainsi, certains, comme Humblé, considèrent que les exemples sont inutiles dans un dictionnaire passif. La définition et l'équivalent de traduction devraient suffire à expliquer le sens d'un mot. Les dictionnaires actifs devraient, au contraire, regorger d'exemples choisis en fonction de leur utilité pour la production de phrases en langue étrangère (Humblé 2001 : 68-69). Dans cet ordre d'idées, il serait préférable que les exemples illustrent des séquences libres, afin d'offrir des modèles pour la production, plutôt que des séquences figées, qui posent surtout des problèmes de compréhension (Humblé 2001: 60).

La place accordée à la traduction dans la dichotomie opposant encoding et decoding n'est pas toujours très claire. Kromann (1987: 59) assimile, d'une part, réception et traduction vers la langue maternelle, et d'autre part, production et traduction vers la langue étrangère. Humblé (2001: 100) attribue deux sens au terme decoding: l'utilisateur peut «décoder» pour comprendre ou pour traduire. L'un ne va pas sans l'autre puisqu'une parfaite compréhension du texte est nécessaire pour la traduction. Le linguiste ajoute alors que l'opération de traduction comporte deux phases: une première phase de decoding et une seconde d'encoding.

Il nous semble que l'exemple a une raison d'être non seulement pour des tâches de production, mais aussi pour la traduction. Toope (1996: 62) s'est penché sur cette question: il distingue les fonctions générales de l'exemple lexicographique, qui valent pour les dictionnaires tant monolingues que bilingues, des fonctions spécifiques de l'exemple du dictionnaire bilingue. Parmi les fonctions générales, Toope (1996) dénombre les fonctions sémantiques, grammaticales et stylistiques. Les fonctions spécifiques se subdivisent, quant à elles, en deux catégories: les fonctions culturelles et les fonctions de traduction. Ces dernières sont particulièrement intéressantes pour la problématique qui nous occupe.

Toope (1996: 96-99) répertorie en fait cinq fonctions de traduction: montrer comment les équivalents sont utilisés dans la langue cible, fournir des équivalents supplémentaires, aider l'utilisateur à choisir l'équivalent le plus approprié au contexte, ajouter des équivalents propres au contexte et enfin suggérer des procédures de traduction indirecte. La première fonction de traduction est essentielle pour les utilisateurs qui effectuent une tâche d'encoding, mais s'avère moins importante pour l'apprenti traducteur dont la langue cible est sa langue maternelle. Les trois fonctions suivantes concernent le choix de l'équivalent le plus adapté. Si cet aspect est bien entendu primordial, il ne sera qu'évoqué rapidement dans la dernière partie de cet article, car nous avons souhaité nous concentrer sur la dernière fonction évoquée par Toope, pour les raisons exposées dans la section suivante.

\section{La traduction de l'exemple comme moyen de suggérer des procédures de traduction indirecte}

Les nuances précises qu'un terme acquiert lorsqu'il est employé en contexte rendent souvent impossible une traduction «directe», mot à mot. Le traducteur doit alors recourir à des procédés tels que la transposition, la modulation, l'expansion ou la paraphrase. Selon Toope (1996: 89), les exemples sont capables d'illustrer les contextes typiques où ces procédés s'imposent et de fournir des pistes de solution. Grundy 
(1996: 148) estime aussi que certaines traductions illustrent des «[...] stratégies qui peuvent être déployées dans des situations qui sont - et nous le découvrons en étudiant le corpus - à la fois fréquentes et problématiques du point de vue de la manipulation syntaxique». Nous sommes convaincue qu'il conviendrait d'inciter les apprentis traducteurs à chercher dans les dictionnaires bilingues plus que de simples équivalents et de les encourager à observer attentivement les traductions des exemples à la recherche de stratégies généralisables qu'ils pourraient mettre en œuvre dans leurs futurs travaux. Nous avons trouvé dans les travaux de Blanco (1995 et 1997) une méthodologie permettant de rendre compte des stratégies visibles dans les traductions des exemples lexicographiques.

Dans sa thèse, Blanco (1995) examine l'ensemble des exemples repris dans les entrées de la lettre $F$ de vingt-huit dictionnaires bilingues espagnol-français et français-espagnol. Pour analyser leur traduction, il distingue dans un premier temps les traductions isomorphes, qu'il définit comme des « séquences $\mathrm{L} 2^{3}$ dont les composants présentent une relation bijective avec ceux de la séquence L1, le sens lexical desdits composants étant semblable dans les deux langues» (Blanco 1997: 134), des traductions hétéromorphes, "[...] séquences L2 dont les composants ne présentent pas une relation bijective avec ceux de la séquence L1 ou si, même en remplissant la condition antérieure, elle contient un composant dont le sens lexical est nettement différent de celui de son homologue en L1 [...]» (Blanco 1997: 135). Autrement dit, les traductions hétéromorphes présentent des divergences de traduction. À de nombreuses occasions, le passage d'une langue à l'autre exige en effet, afin de conserver le même contenu sémantique et d'obtenir un énoncé grammatical en langue cible, que la structure syntaxique superficielle de la séquence cible diffère de celle de la séquence source (Blanco 1995: 934).

Blanco classe ensuite les exemples de son corpus selon la typologie des divergences de traduction créée par Vandooren (1993). Celle-ci distingue différents types de divergences en fonction $\mathrm{du}$ «[...] niveau de description linguistique, à savoir le syntagme, la catégorie morphosyntaxique, la ou les unités lexicales, la position dans la phrase et le prédicat [...]» (Vandooren 1993: 79). Xavier Blanco reprend cette typologie et regroupe donc lui aussi les traductions hétéromorphes en six catégories, selon qu'elles présentent une divergence syntagmatique, catégorielle, lexicale, de densité lexicale (par réduction ou par expansion), thématique ou prédicative.

Blanco soutient que le lexicographe, plutôt que de fournir une traduction isomorphe peu intéressante, peut, grâce à la traduction de l'exemple, mettre en relief certaines différences stylistiques, lexicales ou syntaxiques entre le fonctionnement de L1 et celui de L2, différences imprévisibles si l'on se basait uniquement sur la relation qui unit le lemme à son équivalent de traduction (Blanco 1995: 934). Par conséquent, il faudrait à tout prix éviter que la traduction ne masque les divergences qui existent entre la langue source et la langue cible, mais au contraire chercher à mettre celles-ci en évidence grâce à la traduction (Blanco 1995: 1153).

L'analyse que nous avons menée à bien s'inscrit dans la lignée de celle de Blanco, bien qu'elle ne poursuive pas les mêmes objectifs. Au-delà de la perspective descriptive, la classification des traductions selon les types de divergences qu'elles présentent sert ici de point de départ à une réflexion qui s'articule autour de la question centrale suivante: en quoi le travail du lexicographe facilite-t-il, ou non, la tâche du jeune traducteur qui consulterait le dictionnaire? Lui donne-t-il des outils efficaces pour 
ses traductions futures? Certaines caractéristiques des traductions constituent-elles au contraire des obstacles dans son travail? Les modalités de cette analyse font l'objet de la section suivante.

\section{Modalités de l'analyse : dictionnaires choisis, méthodologie}

Afin de choisir les dictionnaires à analyser, nous avons observé les critères suivants: les ouvrages sélectionnés devaient être des dictionnaires bilingues allemand-français généraux (pas de restriction du vocabulaire) et récents, de grande extension (afin d'augmenter le nombre d'exemples), publiés par des maisons d'édition différentes, toutes très présentes sur le marché. Nous souhaitions également que les ouvrages offrent une grande variété dans leur manière de traiter les exemples, ce dont nous nous sommes assurée par un test préliminaire.

Nous avons finalement retenu trois dictionnaires: le Larousse $^{4}$, le Pons ${ }^{5}$ et le Robert/Collins ${ }^{6}$. Tous trois sont des outils auxquels les étudiants en traduction pourraient réellement avoir recours ${ }^{7}$. Ils présentent cependant des profils divers, tant en ce qui a trait au public visé qu'à l'objectif poursuivi ${ }^{8}$. Le Larousse se présente comme un outil de traduction à destination de lecteurs francophones cultivés, auxquels il souhaite donner un large aperçu du vocabulaire actuel des deux langues. Le Pons, davantage bidirectionnel, insiste sur sa dimension linguistique et sur l'importance de la structuration de ses entrées. Il s'adresse à tous ceux qui font une étude approfondie de la langue allemande. Le Robert/Collins est un outil didactique, conçu pour aider les apprenants, quel que soit leur niveau. Il leur fournit des outils adaptés, en mettant l'accent sur la production.

Comme, en principe, un traducteur travaille au départ d'une langue étrangère vers sa langue maternelle, nous n'avons sélectionné que les exemples donnés en allemand et traduits vers le français, afin de mieux pouvoir juger de l'acceptabilité des traductions et de leur caractère idiomatique. Nous avons choisi de nous intéresser aux verbes, qui offrent souvent des constructions complexes, dont l'analyse devait s'avérer particulièrement riche.

Notre base de données se compose des exemples relevés dans les entrées des dix premiers verbes de chaque lettre de l'alphabet. Elle comprend 942 exemples (479 issus du Larousse, 239 du Pons et 224 du Robert/Collins), accompagnés de leur traduction. Comme les lexicographes ne s'entendent pas sur la définition de l'exemple lexicographique, nous avons recouru à des critères formels et typographiques pour constituer notre corpus. Cependant, il convient de préciser que cette manière de procéder nous a amenée à collecter des éléments de natures diverses. En effet, le Pons structure ses entrées avec rigueur en établissant une distinction entre constructions et locutions idiomatiques. Les deux types de séquences sont pourvus d'exemples d'emploi, qui sont de ce fait pour la plupart des live examples. Dans les deux autres ouvrages, la frontière entre exemples d'emploi et locutions figées est beaucoup plus floue. Par conséquent, certaines séquences que le Larousse et le Robert/Collins présentent comme des exemples sont considérées comme des locutions par le Pons. Les nombres ainsi obtenus montrent que le Robert/Collins fournit en moyenne deux fois plus d'exemples que les deux autres ouvrages'.

La classification des exemples selon la typologie de Vandooren a servi de point de départ à notre analyse. Néanmoins, dans le présent article, nous garderons prin- 
cipalement à l'esprit la distinction fondamentale entre traductions isomorphes et hétéromorphes; les différents types de divergences ne seront évoqués qu'occasionnellement. Nous avons pu constater que, dans les trois dictionnaires, les traductions hétéromorphes étaient beaucoup plus nombreuses (86\%) que les traductions isomorphes, sans doute parce que, d'une part, l'allemand est une langue très différente $\mathrm{du}$ français d'un point de vue structurel, mais aussi, d'autre part, parce que les traductions isomorphes sont réputées inutiles étant donné que l'utilisateur devrait être capable de les produire lui-même dès lors qu'il dispose de l'équivalent. Cette tendance, qui nous a encouragée à nous consacrer en priorité aux divergences de traduction, est encore plus visible dans le Pons, où $93 \%$ des traductions sont hétéromorphes, ce qui pourrait témoigner d'une volonté d'exemplifier en priorité les séquences qui pourraient poser problème à l'utilisateur. Nous reviendrons sur cette orientation du dictionnaire dans la section suivante, dédiée aux questions de linguistique contrastive.

Il convient de préciser que notre objectif n'était pas tant de comparer les dictionnaires, mais plutôt d'observer, dans des ouvrages assez différents, le traitement réservé aux exemples pour déterminer ce qui caractérise les traductions d'exemples lexicographiques potentiellement les plus utiles à l'apprenti traducteur.

\section{Questions de linguistique contrastive}

Blumenthal (1997) compare les systèmes linguistiques allemand et français et met le doigt sur les particularités des deux langues. Son ouvrage nous a permis de déterminer les contrastes sur lesquels les dictionnaires devraient idéalement attirer l'attention de l'utilisateur (Blanco 1995: 1153) pour lui suggérer des solutions de traduction potentiellement généralisables, qu'il pourrait mettre en œuvre dans des tâches de traduction (Toope 1996: 89).

Les exemples repris ci-dessous illustrent cette fonction de l'exemple du dictionnaire bilingue. Nous nous pencherons sur les phénomènes suivants, choisis parmi d'autres questions de linguistique contrastive: la traduction de la voix passive, de la notion d'espace (en particulier, des prépositions spatiales et des verbes de mouvement), des déterminants, des auxiliaires de mode, des particules modales et de l'infinitif substantivé.

Selon Blumenthal, une phrase est d'autant plus active que sa formulation correspond au schéma agens - actio - patiens. Il soutient que, dans de nombreuses constructions typiques des deux langues, la phrase française exprime un degré d'activité plus élevé que la phrase allemande (Blumenthal 1997: 17). L'allemand évoquerait plus souvent des processus ou des états alors que le français préférerait les actes. Étant donné la fréquence plus élevée de tournures passives en allemand, opter systématiquement pour une tournure passive en français n'est pas la solution idéale. Le traducteur est donc amené à envisager d'autres possibilités de traduction, hétéromorphes. Les dictionnaires reflètent cet état de fait puisque, dans la majorité des traductions observées, la voix active remplace en français la voix passive allemande. Se pose alors la question du sujet: soit il opte pour le pronom indéfini on, soit pour un sujet général.

1) etw wird zu Mehl gemahlen - on moud qc pour en faire de la farine

(Pons 2000: 443; nous soulignons)

2) überall wird wild gecampt - tout le monde fait du camping sauvage

(Pons 2000: 149; nous soulignons) 
Une autre solution est de faire de l'agent le sujet grammatical de la phrase.

3) etw kann nicht mehr länger von jdm. ignoriert werden - qn ne peut plus ne pas tenir compte plus longtemps de qc.

(Pons 2000: 364; nous soulignons)

Blumenthal (1997: 63, 67) montre également que l'allemand prête davantage attention à la notion d'espace que la langue française et dispose de moyens plus diversifiés pour l'exprimer. L'allemand peut en effet indiquer la direction, l'origine ou encore la situation d'une personne ou d'un objet de manière plus précise que le français, et ce, non seulement grâce à l'opposition entre l'accusatif et le datif, mais aussi grâce à ses préfixes verbaux et à ses prépositions. Les prépositions françaises ont un sens moins précis que les prépositions allemandes et sont davantage polysémiques (Blumenthal 1997: 66). Comme elles ne suffisent pas à exprimer l'idée de mouvement, en français, c'est le verbe qui doit jouer ce rôle, c'est pourquoi, dans certains cas, la traduction introduit une divergence prédicative ${ }^{10}$ par rapport à l'exemple allemand. L'accent est alors déplacé: alors qu'en allemand, le verbe exprime la manière de se déplacer et la préposition exprime le mouvement, l'inverse se produit en français.

4) die Reiter galoppieren über das Feld - les cavaliers traversent le champ au galop

(Pons 2000: 271; nous soulignons)

5) jd quält sich auf den Berg - qn escalade péniblement la montagne

(Pons 2000: 519; nous soulignons)

Par ailleurs, en allemand, certains verbes fonctionnent par paire: l'un indique le mouvement et l'autre la situation. Il en va de la sorte pour setzen [mouvement] et sitzen [situation]. Il est parfois difficile - et pas toujours souhaitable - de rendre cette différence perceptible dans la traduction, c'est pourquoi le Pons (2000: 313) précise, juste avant les exemples concernés que das Verb wird oft nicht übersetzt.

6) ich habe noch Gäste im Wohnzimmer sitzen - j'ai encore des invités [qui attendent] au salon

(Pons 2000: 313; nous soulignons)

Le verbe sitzen employé dans l'exemple 6 indique deux choses: d'une part, que les invités sont assis, d'autre part, qu'ils ne se déplacent pas. La première idée n'est pas clairement exprimée en français, mais peut être déduite du contexte. La deuxième est rendue par qui attendent, mais, comme le montre la présence des crochets, la phrase française pourrait très bien (voire mieux) fonctionner sans cette précision. Certaines informations sont donc perdues dans la traduction, mais les expliciter toutes s'avérerait sans doute incongru et peu conforme à l'usage.

L'omission semble également s'imposer pour certains auxiliaires de mode et certaines particules modales. Alors que le français ne compte que trois auxiliaires de mode (vouloir, pouvoir et devoir), l'allemand en a six: wollen, sollen, dürfen, müssen, können et mögen (Malblanc 1944/1968: 267). Selon Blumenthal (1997: 15), les francophones ressentiraient souvent l'emploi d'une modalisation comme une spécification inutile et dérangeante. Dans de nombreux exemples, l'auxiliaire de mode est omis dans la traduction française. 
7) machen Sie, dass ich nie wieder von Ihnen hören muss! - arrangez-vous pour que [o faites en sorte que] je n'entende plus jamais parler de vous!

(Pons 2000: 441; nous soulignons)

La même solution semble privilégiée pour traduire les particules modales. Traditionnellement, les linguistes en dénombrent 18 en allemand: $\underline{\text { aber, }} \underline{\text { auch, }} \underline{\text { bloß }}$, denn, doch, eben, eigentlich, einfach, etwa, erst, halt, ja, mal, nur, ruhig, schon, vielleicht et wohl (Blumenthal 1997: 92). En employant ces particules, le locuteur laisse paraître sa subjectivité, son attitude par rapport à son propre discours. Longtemps perçues comme des mots «bouche-trous» indésirables, ces particules sont aujourd'hui considérées comme une véritable richesse de la langue allemande. Le français possède des particules remplissant des fonctions similaires (bien, donc, peut-être, déjà, mais). Toutefois, celles-ci sont, d'une part, beaucoup moins nombreuses et, d'autre part, moins fréquemment utilisées que les particules modales allemandes. Les francophones ont en effet recours à d'autres moyens pour exprimer leur subjectivitél ${ }^{1}$. Selon Blumenthal (1997: 93), la spécificité de la langue allemande n’est donc pas tant de disposer de moyens de modaliser un énoncé, mais plutôt de concentrer l'expression de la subjectivité sur une classe de mots.

Puisque cette classe n'existe pas comme telle en français, la traduction des particules modales n'est pas toujours aisée. Le traducteur peut, bien sûr, recourir aux particules susmentionnées ou reproduire le sens et l'effet de la particule modale allemande grâce à des interjections, par exemple. Toutefois, dans la majorité des cas, la particule n'est pas traduite (Blumenthal 1997: 95). L'analyse des dictionnaires envisagés rejoint les observations du linguiste. Dans de rares cas, la particule modale est traduite par une particule en français.

8) da kann man wohl sagen, daß - c'est bien le cas de dire que

(Larousse 2007: 649; nous soulignons)

Dans d'autres cas, le sens de la particule modale est rendu d'une autre façon:

9) hast du mir mal ne Zigarette? - tu n'aurais pas une cigarette?

(Pons 2000: 313; nous soulignons)

Dans cet exemple, la particule mal sert à atténuer la force de l'interrogation, effet reproduit en français grâce à la négation et à l'emploi du conditionnel. Néanmoins, dans la grande majorité des cas, la particule modale n'est pas traduite, comme dans l'exemple 10.

10) FAM. mach doch keine Geschichten! - ne fais pas d'histoires!

(Larousse 2007: 522; nous soulignons)

On retrouve donc au sein des dictionnaires la différence de fréquence d'utilisation des particules modales entre l'allemand et le français relevée par Blumenthal.

Nous avons relevé de nombreux exemples dont la traduction différait de l'original uniquement par l'article utilisé. Ces exemples pourraient contribuer à attirer l'attention de l'utilisateur sur le risque de ce que Blumenthal (1997: 79-80) appelle Überbestimmung [sur-détermination] ou Unterbestimmung [sous-détermination]. Le linguiste met notamment le doigt sur la tendance de l'allemand à utiliser l'article défini, même pour des éléments encore inconnus du lecteur qu'une lecture attentive permettrait de deviner. Selon lui, le traducteur français, qui adopte la position du 
lecteur, opterait le plus souvent pour un article indéfini (Blumenthal 1997: 78). L'exemple 11 illustre cette tendance:

11) tief gähnt der Abgrund vor ihnen - un précipice géant s'ouvre devant eux

(Larousse 2007: 307; nous soulignons)

D’autres exemples témoignent de la différence d'utilisation des déterminants démonstratifs et possessifs en français et en allemand. Le cas le plus fréquent est celui où un article défini allemand est traduit par un démonstratif en français.

12) der neue Wagen fährt sich sehr angenehm - cette nouvelle voiture a une conduite très agréable

(Pons 2000: 238; nous soulignons)

En réalité, l’article défini a, en allemand, une valeur déictique (Malblanc 1944/1968: 260) que la traduction rend ici explicite. La même tendance peut être observée pour le déterminant possessif, plus souvent employé en français dans notre corpus:

13) einen Beitrag zahlen - verser sa cotisation

(Larousse 2007: 929; nous soulignons)

Aussi bien en français qu'en allemand, il est possible de nominaliser les infinitifs. Néanmoins, l'emploi de l'infinitif substantivé est beaucoup plus fréquent en allemand (Malblanc 1944/1968: 86). Le traducteur francophone peut donc éprouver certaines difficultés à le traduire de manière adéquate. C'est peut-être pour cette raison que le Pons intègre systématiquement l'infinitif substantivé à sa microstructure. Le dictionnaire s'attache à en illustrer l'usage avec différentes prépositions et accorde une attention particulière à l'infinitif substantivé précédé de bei, qui représente sans nul doute une difficulté de traduction.

14) P161: störe mich nicht beim Häkeln - ne me dérange pas quand je fais du crochet

(Pons 2000: 315; nous soulignons)

Le dictionnaire fait systématiquement correspondre cette construction à une proposition temporelle introduite par quand, une solution que l'utilisateur pourrait adopter dans ses propres traductions.

Les traductions dictionnairiques illustrent donc bel et bien certaines stratégies de traduction, certaines «routines des traducteurs professionnels» (Humblé 2010: 336) desquelles l'utilisateur pourrait s'inspirer pour surmonter les obstacles posés par les différences syntaxiques entre deux systèmes linguistiques. Si ces stratégies peuvent être observées dans les trois dictionnaires, le grand nombre d'exemples cités dans cette section et issus du Pons témoigne de l'attention particulière que l'ouvrage accorde aux questions de linguistique contrastive. Ce dictionnaire est par ailleurs le seul à illustrer certains phénomènes linguistiques.

\section{Traductions discutables}

L'analyse de notre corpus d'exemples a permis de déceler un certain nombre de traductions discutables. Certaines s'avèrent "inutilisables» telles quelles et, dès lors, insuffisantes: elles nécessitent une intervention de l'utilisateur, qui devra fournir un effort pour compléter lui-même une construction grammaticale ou pour trouver les informations manquantes dans d'autres ouvrages. D'autres pourraient induire l'uti- 
lisateur en erreur quant au registre auquel appartient une expression, au contexte dans lequel il convient de l'employer ou même quant au sens qu'il convient de lui attribuer.

Un problème se pose lorsque les exemples présentent une omission, c'est-à-dire lorsqu'un élément présent dans la construction allemande n’apparaît pas en français. $\mathrm{Si}$, dans certains cas, la suppression de cet élément est volontaire et vise à alléger la traduction, dans d'autres, l'omission est plus problématique.

15) über etwas nachdenken - gamberger

(Larousse 2007: 552; nous soulignons)

L’utilisateur pourrait ici hésiter quant à la préposition qui régit le complément du verbe gamberger et se verrait donc obligé de recourir à une autre source d'information.

L'exemple suivant s'avère lui aussi problématique:

16) fahr ruhig mal [mit der Hand/dem Tuch] darüber - passe ta main/le chiffon dessus

(Pons 2000: 238)

Si l'on excepte les syntagmes facultatifs de l'expression illustrée dans l'exemple 16, l'exemple se limite à fahr ruhig mal darüber. Toutefois, il est difficile de trouver la traduction de cette construction minimale, puisque les éléments facultatifs sont intégrés à la traduction proposée, cette fois-ci sans crochets. Or, traduire fahr ruhig mal darüber par passe dessus serait peu idiomatique. Les informations fournies par le dictionnaire ne sont donc pas toujours suffisantes.

Pour le traducteur, il est important de connaître le registre d'une expression afin de choisir le meilleur équivalent possible en français. Malheureusement, de ce point de vue non plus, les dictionnaires bilingues ne sont pas toujours rigoureux et, dans de nombreux cas, les exemples ne comportent aucune indication de registre. En l'absence de précisions, l'utilisateur partira du principe que la traduction relève d'un registre équivalent à celui de l'exemple allemand (Blanco 1995: 1118), alors que ce n'est pas toujours le cas. En effet, le Larousse, notamment, propose à plusieurs reprises des traductions dont le registre ne semble pas correspondre à celui de l'expression originale en allemand.

17) jn zum besten haben - se jouer de, mystifier qn; FAM. mener qn en bateau

(Larousse 2007: 367)

Le Larousse propose trois traductions différentes pour l'exemple 17. Entre les deux premières, qui ne sont précédées d'aucune indication de registre, et la dernière, dont le dictionnaire précise qu'elle est familière, l'écart est énorme. Le francophone est capable de percevoir cette différence. Néanmoins, le dictionnaire ne lui fournit aucune information quant au registre de l'expression allemande. Selon le Duden ${ }^{12}$, jn zum besten haben est une expression familière, ce qui fait de la troisième traduction $\mathrm{du}$ Larousse, mener qn en bateau, une bonne traduction. Les deux autres, en revanche, sont en décalage par rapport à l'allemand, ce dont l'utilisateur pourrait ne pas se rendre compte.

En outre, de nombreux exemples proposés par le Larousse sont traduits par des expressions vieillies, qui ne correspondent pas à l'usage actuel de la langue, alors que les expressions allemandes correspondantes ne le sont pas.

18) das garantiere ich dir - je te donne ma parole; FAM., POP. je te fiche mon billet

(Larousse 2007: 310) 
19) sich krank lachen - être malade de rire; se pâmer de rire

20) auf jn zählen - compter, faire fond sur qn

(Larousse 2007: 929)

En effet, Le Petit Robert ${ }^{13}$ considère l'expression je vous fiche mon billet, employée pour traduire l'exemple 18, comme vieillie. Ce dictionnaire qualifie également de vieilli le sens "perdre connaissance» du verbe pâmer, employé dans l'exemple 19. Il en va de même pour la traduction de l'exemple 20, locution verbale faire fond sur, considérée comme vieillie par le $T L F i^{14}$. Il convient par ailleurs de noter que, dans les trois cas susmentionnés, la traduction datée suit une traduction plus actuelle, ce qui nous permet de douter de son utilité. Dans certains cas extrêmes, le caractère daté de la traduction retenue pourrait même entraver la bonne compréhension de l'expression allemande par un jeune traducteur francophone.

La question du sens est évidemment fondamentale dans une perspective de traduction. Dans certains cas, pourtant, le sens de la traduction s'écarte de celui de l'original.

21) sich einen guten Tag machen - paresser

(Larousse 2007: 522)

La traduction proposée par le Larousse pour l'exemple 21, paresser, est réductrice. En effet, il ne s'agit pas uniquement de «se laisser aller à la paresse, à l'oisiveté; ne rien faire» (Le Petit Robert), mais plutôt de s'octroyer quelque chose d'agréable (voir Duden Band $11^{15}$ : 710).

22) sich das Leben leicht machen - prendre la vie du bon côté

(Larousse 2007: 522)

Dans le même ordre d'idées, la traduction de l'exemple 22 ne correspond pas exactement à l'original allemand. Prendre la vie du bon côté signifie «envisager [la vie] sous un jour favorable, profiter des plaisirs de l'existence» (TLFi). Or, l'expression allemande signifie plutôt «se faciliter la vie» et, dans certains contextes, peut être employée de manière péjorative («choisir la solution de facilité »).

Dans d'autres cas, la traduction française proposée par les dictionnaires est ambiguë: l'expression française est trop vague ou polysémique pour que l'utilisateur puisse l'interpréter et comprendre le véritable sens de l'exemple allemand.

23) dich hats wohl! (umg) - ça va pas?

(Robert 2012: 177)

Lorsqu'il prononce la phrase 23 dich hats wohl, le locuteur ne s'inquiète pas de la santé ou des états d'âme de son interlocuteur. Au contraire, il lui fait remarquer qu'il se montre déraisonnable. La traduction proposée ne permet pas au francophone de le comprendre. Une traduction légèrement différente, comme ça va pas la tête?!, aurait permis d'éviter cette ambiguïté.

24) er wird es nicht mehr lange machen - il n'ira pas loin

(Larousse 2007: 521)

L'expression er wird es nicht mehr lange machen signifie que la personne en question va bientôt mourir (voir Duden). Néanmoins, il serait surprenant que l'uti- 
lisateur interprète la traduction française dans ce sens. Une traduction du type il ne fera plus long feu ou il n'en a plus pour longtemps aurait mieux convenu.

25) jn über den Haufen fahren - culbuter qn

(Larousse 2007: 264)

Dans l'exemple 25, le francophone risque bien de ne pas savoir quel sens attribuer à culbuter. Le choix d'un complément humain (jn) ne facilite pas l'interprétation, qui penchera facilement vers "renverser [une femme] pour la posséder charnellement» plutôt que vers «faire tomber en renversant ou en poussant brutalement» (TLFi). Or, ce deuxième sens est le seul que peut avoir l'expression allemande (voir Duden).

Il est également important que la proposition de traduction puisse être utilisée sous les mêmes conditions que l'original. L'exemple suivant montre qu'il n'en va pas toujours ainsi.

26) mit Verlaub zu sagen - s'il est permis de dire (que)

(Larousse 2007: 649)

En allemand, l'expression mit Verlaub (zu sagen) est employée en incise, comme dans les phrases suivantes: Das ist dem - mit Verlaub, Herr Hauptmann - scheißegal! et Und wie ich, mit Verlaub, mein Geschäft erledigt habe, merke ich: Da ist kein Klosettpapier (voir Duden Band 11: 760). Il n'est pas possible d'utiliser la traduction proposée par le Larousse pour l'exemple 26 de la même manière. L'utilisateur éprouvera donc des difficultés à employer la solution fournie par le dictionnaire dans un cotexte similaire au cotexte allemand. Une traduction du type si je puis dire ou passez-moi l'expression réglerait ce problème.

\section{Conclusions}

L'analyse des exemples lexicographiques issus du Larousse, du Pons et du Robert/ Collins a permis de mettre en lumière certaines caractéristiques des exemples les plus utiles au jeune traducteur. Nous l'avons vu, Xavier Blanco (1995: 1155) affirme que la traduction des exemples lexicographiques devrait mettre en évidence les différences existant entre deux systèmes linguistiques. Étant donné la proportion importante de traductions hétéromorphes au sein de notre corpus, il nous semble que ces différences justifient en effet la présence des exemples dans un ouvrage. Nous espérons avoir montré que les dictionnaires bilingues illustrent bel et bien les grandes tendances de la linguistique contrastive allemand-français. C'est particulièrement vrai pour le Pons, qui adopte une perspective linguistique clairement perceptible. Malheureusement, les exemples d'une telle qualité contrastive sont aujourd'hui encore trop rares.

En attirant l'attention de l'utilisateur sur lesdites différences, les exemples pourraient l'inciter à ne pas opter systématiquement pour une traduction isomorphe et lui proposer une solution alternative. Toutefois, nous nous permettons d'émettre quelques réserves quant à la fonction de traduction décrite par Michael Toope (1996). Si la traduction d'un exemple, prise de manière isolée, peut en effet suggérer une procédure de traduction indirecte dans un cas précis, l'utilisateur pourrait ne pas s'apercevoir qu'elle a valeur générale. En effet, pour déduire des règles généralisables et applicables dans une tâche de traduction, il devrait fournir un certain travail de systématisation, effort qu'il n'est pas forcément prêt à consentir. 
Par ailleurs, nous avons pu déceler dans notre échantillon un certain nombre de traductions discutables. Nos observations nous permettent de suggérer quelques pistes d'amélioration pour remédier à ces lacunes. Pour être réellement utile à l'utilisateur, un dictionnaire devrait traduire tout l'exemple et rien que l'exemple, c'està-dire éviter les omissions et les ajouts, sous peine de forcer l'utilisateur à recourir à une seconde source d'information. De plus, le registre de l'expression originale devrait être clairement indiqué afin de guider le choix du traducteur. Puisque la traduction passe d'abord par une parfaite compréhension du texte, les traductions proposées ne devraient permettre aucun doute quant au sens de l'expression originale. Elles devraient en outre correspondre à l'usage actuel de la langue.

\section{NOTES}

1. Les traducteurs confirmés se plaignent en effet que les dictionnaires bilingues ne recensent pas les emplois les plus rares (Grundy 1996: 147). Ils n’y trouvent pas toujours les réponses à leurs interrogations, qui vont bien au-delà de l'aspect purement lexical et sont très souvent extrêmement dépendantes du contexte (Varantola 1998: 180-181).

2. Selon Atkins et Rundell (2008: 465), le travail sur un corpus comporte trois phases: analyse, transfert et synthèse.

3. Dans ses définitions, Xavier Blanco (1997: 134-135) emploie l'expression «séquence L1 » pour faire référence à l'exemple et «séquence L2» pour designer la traduction de l'exemple.

4. Grappin, Pierre, dir. (2007): Grand dictionnaire allemand-français, français-allemand = Grosswörterbuch Deutsch-Französisch, Französisch-Deutsch. Paris: Larousse.

5. SCHNORR, Veronika, dir. (2000): Pons Grosswörterbuch für Experten und Universität: FranzösischDeutsch, Deutsch-Französisch [Le Grand Pons pour les experts et l'université: français-allemand, allemand-français]. Stuttgart/Düsseldorf: Ernst Klett.

6. BeAttle, Susie, dir. (2012): Le Robert \& Collins allemand maxi: français-allemand, allemandfrançais. Paris/Glasgow: Le Robert/Collins.

7. Ils sont par exemple disponibles à la bibliothèque de l'Université de Liège, où nous avons mené notre étude.

8. Les informations suivantes, relatives au public visé et à l'objectif poursuivi par les dictionnaires, sont issues des préfaces de chacun des ouvrages.

9. Le cas du verbe machen attire particulièrement l'attention: le Larousse n'en donne pas moins de 131 exemples d'emploi, le Pons, 60 et le Robert/Collins, 43 seulement. Dans le Larousse, la multiplication des exemples rend parfois les entrées difficiles à consulter.

10. «On parle de divergence prédicative quand le prédicat est différent en LS [langue source] et en LC [langue cible]; la réalisation du prédicat est inverse entre le verbe principal et un complément.» (Vandooren 1993: 81). Les exemples de divergence prédicative donnés par Vandooren pour les verbes de mouvement sont similaires à ceux que nous avons relevés dans les dictionnaires.

11. L'intonation, notamment, joue un rôle majeur.

12. Duden Online. Leipzig: Bibliographisches Institut. Consulté le 17 mars 2018, <http://www.duden. de/woerterbuch>.

13. Rey-Debove, Josette et Rey, Alain, dir. (2008): Le nouveau Petit Robert. Paris: Le Robert.

14. Analyse et Traitement Informatique de la Langue Française (Atilf) (1994): Trésor de la langue française informatisé (TLFi). Nancy: Université de Lorraine. Consulté le 1 mars 2018, $<$ http://atilf.atilf.fr $>$.

15. Drosdowski, Günther et Scholze-Stubenrecht, Werner, dir. (1992): Redewendungen und sprichtwörtliche Redensarten. Duden Band 11 [Expressions idiomatiques et proverbiales. Duden volume 11]. Mannheim/Leipzig/Vienne/Zurich: Dudenverlag.

\section{RÉFÉRENCES}

Atkins, B. T. Sue et Rundell, Michael (2008): The translation stage. In: B. T. Sue Atkins et Michael Rundell. The Oxford Guide to Practical Lexicography. Oxford: Oxford University Press, 465-483. 
Blanco, Xavier (1995): El ejemplo en el diccionario bilingüe. Tipología y funciones del ejemplo en el marco de la lexicografía bilingüe general contemporánea francés-español, españolfrancés. Thèse de doctorat, non publiée. Barcelone: Universitat Autónoma de Barcelona.

Blanco, Xavier (1997): Lexicographie bilingue (français-espagnol) et traduction: l'exemple. Meta. 42(1):133-141.

Blumenthal, Peter (1997): Sprachvergleich Deutsch-Französisch [Étude comparée des langues allemande et française]. Tubingue: Max Niemeyer.

Grundy, Valérie (1996): L'utilisation d'un corpus dans la fabrication des dictionnaires bilingues. In: Henri BÉJoInt et Philippe Thorron, dir. Les dictionnaires bilingues. Louvain-la-Neuve: Duculot, 127-149.

Humblé, Philippe (2001): Dictionaries and Language Learners. Francfort-sur-le-Main: Haag und Herchen.

Humblé, Philippe (2010) : Dictionnaires et traductologie: le paradoxe d'une lointaine proximité. Meta. 55(2):329-337.

Jacobsen, Jans Rosenkilde, Manley, James et Pedersen, Viggo Hjørnager (1991): Examples in the bilingual dictionary. In: Franz Josef Hausmann, Oskar Reichmann, Herbert Ernst Wiegand, et al., dir. Wörterbucher / Dictionaries / Dictionnaires. Berlin/New York: de Gruyter, 2782-2789.

Kosem, Iztok (2016): Interrogating a corpus. In: Philip Durkin, dir. The Oxford Handbook of Lexicography. Oxford: Oxford University Press, 76-93.

Kromann, Hans-Peder (1987): Neue Orientierung der zweisprachigen Wörterbücher. Zur funktionalen zweisprachigen Lexikographie [Nouvelle orientation des dictionnaires bilingues. De la lexicographie bilingue fonctionnelle]. In: Mary SNELL-Hornby et Esther PöHL, dir. Translation and Lexicography. Amsterdam/Philadelphie: John Benjamins, 55-65.

Mackintosh, Kristen (1998): An Empirical Study of Dictionary Use in L2-L1 Translation. In: B. T. Sue AtKins, dir. Using dictionaries: studies of dictionary use by language learners and translators. Tubingue: Max Niemeyer, 123-149.

Malblanc, Alfred (1944/1968): Stylistique comparée du français et de l'allemand. Paris: Didier.

Martin, Robert (1989): L'exemple lexicographique dans le dictionnaire monolingue. In: Franz Josef Hausmann, Oskar Reichmann, Herbert Ernst Wiegand et al., dir. Wörterbücher/ Dictionaries/Dictionnaires. Berlin/New York: de Gruyter, 599-607.

Rettig, Wolfgang (1985): Die zweisprachige Lexikographie Französich-Deutsch, DeutschFranzösisch. Stand, Probleme, Aufgaben [La lexicographie bilingue français-allemand, allemand-français. État de la question, problèmes, tâches futures]. Lexicographica. 1:83-125.

Starren, Peter et Thelen, Marcel (1990): General Dictionaries and Students of Translation: A Report on the Use of Dictionaries in the Translation Process. In: Tamás MagaY et Judit ZIGANY, dir. Papers from the Third International EURALEX Congress. Budapest: Akademiai Kiado, 447-458.

Svensén, Bo (1993): Practical lexicography. Principles and Methods of Dictionary-Making. Oxford/New York: Oxford University Press.

Tognini-Bonelli, Elena (2001): Corpus Linguistics at Work. Amsterdam/Philadelphie: John Benjamins.

Toope, Michael (1996): Examples in the Bilingual Dictionary. Mémoire de maîtrise, non publié. Ottawa: Université d'Ottawa.

VAndooren, Françoise (1993): Divergences de traduction et architectures de transfert. In: Pierre Boulllon et André Clas, dir. La traductique. Montréal: Presses de l'Université de Montréal, 77-90.

Varantola, Krista (1998): Translators and their Use of Dictionaries: User Needs and User Habits. In: B. T. Sue AtKins, dir. Using dictionaries: studies of dictionary use by language learners and translators. Tubingue: Max Niemeyer, 179-192. 